\title{
O sistema social da saúde: conceito, limites e possibilidades
}

The social system of health: concept, limits and possibilities

\section{Sandra Regina Martini Vial}

Pós-doutora em Direito. Professora da Universidade do Vale do Rio dos Sinos (Brasil), da Scuola Dottorale Internazionale Tullio Ascarelli e professora visitante da Università Degli Studi di Salerno (Itália).

Resumo: Discutem-se as possibilidades de definir o sistema da saúde como um sistema social, diferenciado funcionalmente, a partir da ótica da Teoria dos Sistemas Sociais, utilizando os pressupostos gerais da Teoria e a estrutura apresentada por Niklas Luhmann sobre o sistema médico. Apresentam-se alguns "desassossegos" provocados pela Teoria; as possíveis relações entre o sistema da saúde e o que Luhmann chamou de sistema médico e como esse sistema, atualmente, pode ser considerado sistema da saúde; e, por fim, a nossa concepção do sistema da saúde na atualidade.

Palavras-chave: sistema da saúde; sistema médico; direito à saúde.

Resumen: Se discuten las posibilidades de definir el sistema de la salud como un sistema social, funcionalmente diferenciado, desde la perspectiva de la Teoría de los Sistemas Sociales, utilizando los presupuestos generales de la teoría y la estructura presentada por Niklas Luhmann sobre el sistema médico. Presentan se las "inquietudes" provocadas por la teoría; las posibles relaciones entre el sistema de la salud y el que Luhmann denomina sistema médico y de cómo este sistema, en la actualidad, se puede considerar como el sistema de la salud; y, por último, se presenta nuestra concepción del sistema de atención a la salud hoy en día.

Palabras clave: Sistema de salud; sistema médico; derecho a la salud.

Abstract: The article presents the possibilities to define the social system of health as a social system, functionally differentiated, from the perspective of the Social Systems Theory. To achieve this goals, we're going to use the main assumptions of the Theory and the structure explained by Niklas Luhmann about the medical system. First, we're going to presents some "unrests" caused by the Theory; next, the possible relationships between the medical system and the current system of healthcare; then, we're going to explain our conception about the social system of healthcare nowadays.

Key-words: system of health; medical system; right to healthcare. 


\section{Introdução}

Le transformazione semantiche seguono le transformazione strutturali a considerevole distanza. Perchè, attraverso il ripetere e il dimenticare, possa condensarsi senso a condizione di tipo nuovo, se richiede tempo. È nostra impressione che, riguardo a ciò, la società moderna sia solo all'inizio. L'evidente insoddisfazione per tuto ciò che atualmente viene offerto, potrebbe diventare un inizio più fecondo ${ }^{1}$. (Luhmann; De Giorgi, 1996, p.400).

As transformações da sociedade atual são maiores do que podemos prever, são mais profundas e mais rápidas do que em qualquer outro momento histórico. Por isso, é necessário repetir e esquecer para assim entender o novo do novo, ou o velho do novo e o novo do velho.

Realmente estamos apenas no início de uma sociedade altamente complexa. Esse cenário - novo/velho - apresenta-se como desafio para novas análises, estudos e pesquisas. As teorias têm mostrado suas limitações, mas também suas possibilidades, e estas - as possibilidades -, muitas vezes, são visíveis somente através das limitações. É nesse jogo que precisamos ousar. Ousar a partir de teorias que desafiam certezas e que propõem uma análise mais fecunda para esta sociedade que está apenas no início, na qual o presente deve ser entendido como diferença entre futuro e passado. Assim, temos um problema com relação à certeza, o que acaba estimulando o desenvolvimento de uma dimensão temporal das experiências vividas e das ações. É neste tempo que se deve responder tanto à certeza como à incerteza em relação às expectativas sociais que são continuadamente frustradas/satisfeitas por meio da complexidade social em permanente incremento.

As teorias ou as ciências produzem efeitos diversos para quem se debruça seriamente sobre o estudo das condições de utilizá-las na análise dos fenômenos sociais. No caso específico da Teoria dos Sistemas Sociais de Niklas Luhmann, esse efeito se transforma em "desassossego" porque rompe com pressupostos e verdades, tornando isto complexo e desafiador. Essa Teoria se apresenta com vários diferenciais, pois, além de desacomodar qualquer pesquisador, também se apresenta como um desafio para a análise da complexidade social, uma vez que não aceita as

\footnotetext{
1 "As transformações semânticas seguem as transformações estruturais a uma considerável distância. Porque, por meio da repetição e do esquecimento, possa condensar-se no sentido de condicionar a tipos novos, requer tempo. É a nossa impressão que, no que diz respeito a isso, a sociedade moderna esteja somente no início. A evidente insatisfação para tudo aquilo que é oferecido, poderia se tornar um início mais frutífero". Tradução livre.
} 
teorias tradicionais na sua íntegra, mas as utiliza para mostrar as fragilidades internas da própria Teoria.

Toda a base de construção da Teoria é transdisciplinar e construtivista. Isso, por um lado, facilita o processo de compreensão da sociedade atual, mas, por outro lado, não admite análises lineares, ou seja, cada análise pode ser diferente da que foi formulada.

Esses desassossegos serão tratados no início do artigo, recordando que o próprio Niklas Luhmann (Luhmann; De Giorgi, 1996) referia a importância dessas inquietações, como outros autores também vêm advertindo para a importância deste aspecto, sem o qual não se pode entender a complexidade da Teoria. A seguir, trataremos da possibilidade de definir a saúde como um sistema social, diferenciado funcionalmente.

Muito embora o autor não tenha trabalhado diretamente com o sistema da saúde, mas sim com o sistema médico, vemos vários elementos da Teoria que nos dão bases para defender a postura de que a saúde é um sistema social. Por exemplo, o fato de que, constantemente, recebe irritações ${ }^{2}$ de outros sistemas sociais, em especial - ou talvez a mais conhecida - a irritação do sistema do direito. A materialização dessa irritação pode ser percebida como a "judicialização da saúde", processo em que o Poder Judiciário atua para tutelar a efetividade do direito à saúde. O apogeu desse processo ocorreu no final da década de 1990, época em que foi possível observar que mais de $50 \%$ das demandas judiciais estavam vinculadas à saúde. Em outras palavras, podemos ver claramente a relação entre dois sistemas sociais, cada um operando com uma estrutura interna específica e constantemente produzindo irritações recíprocas.

Nesse período de contato entre sistemas sociais, o Direito Sanitário consolidou-se como linha de pesquisa em diversos centros acadêmicos e profissionais, provocando muitas indagações, o que é típico de uma nova ciência que começa a se solidificar. A ciência que não provoca este tipo de desafio certamente não toma posse da realidade, ou melhor, não entende que tomar posse da realidade é diferenciar sistema e ambiente, é entender a sociedade como a diferença constitutiva entre sistema e ambiente.

\footnotetext{
${ }^{2}$ Para a Teoria dos Sistemas Sociais, irritação significa o constante contato entre um sistema social e outro.
} 
Tomar posse da realidade significa buscar constantemente o desvelamento de paradoxos, sabendo que esse também é um processo circular: quando desvelamos um paradoxo, corremos o risco de esconder outros. Portanto, a necessidade de entender a sociedade como um sistema em evolução é fundamental e, mais do que isso, é preciso entender que a evolução é um processo que não podemos controlar. Ademais, só temos evolução quando o sistema decide por algo pelo qual anteriormente não se poderia decidir.

Este artigo será fundado em várias inquietações e reflexões sobre a ideia de sistema. Especificamente, pretendemos fundamentar que a saúde pode ser entendida no contexto de um sistema social diferenciado, fazendo parte desta grande rede ou malha de comunicações chamada sociedade. Além das reflexões teóricas a respeito da possiblidade de limitações da definição do "sistema da saúde" como sistema, também temos outras indagações desde a perspectiva sociojurídica, como a redefinição conceitual de 'mudança social' e de 'transformação social' em uma sociedade complexa, paradoxal, na qual o processo evolutivo nos "obriga" a definir e redefinir constantemente os conceitos construídos. Não poucas vezes, esta construção se dá através da desconstrução, postura típica da Teoria Sistêmica, a qual nos permite refletir e questionar o processo atual de transformação social. Por isso, a teoria "desassossega" e "molesta".

\section{Os desassossegos da Teoria: limites e possibilidades para ultrapassar e criar a partir da Teoria Sistêmica.}

Oggetto delle nostre ricerche è il sistema sociale della soceità moderna. Intendiamo realizzare un progetto che incontra numerose difficoltà. La prima è costituita dal progetto stesso, il quale attualizza un rapporto circolare con il suo oggetto: un oggetto che non ha referenze univoche ${ }^{3}$. (Luhmann; De Giorgi, 1996, p. 9)

Os desassossegos foram discutidos por Luhmann (Luhmann; De Giorgi, 1996), como podemos perceber na passagem acima, quando afirmava que o projeto que pretendia encontrava dificuldades iniciais na definição do próprio projeto; mais do que isso, ele propunha a eliminação da diferença sujeito-objeto. Proposta já apresentada

\footnotetext{
3 "O objeto de nossas pesquisas é o Sistema Social da sociedade moderna. Pretendemos realizar um projeto que encontra inúmeras dificuldades. A primeira é constituída pelo projeto em si, o qual atualizada uma relação circular com o seu objeto: um objeto que não há referências únicas". Tradução Livre.
} 
por outras teorias como, por exemplo, pelas teorias marxistas ${ }^{4}$, que afirmam não haver a possibilidade desta separação, mas não criam novas categorias para resolver a complexidade dessa situação.

A Teoria Sistêmica propõe a distinção sistema-ambiente, não com a pretensão de resolver a complexidade, mas de desvelá-la. Fazer esta operação significa tentar definir o social e a própria sociedade, tarefa enfrentada por Luhmann em todos os textos e reflexões, porém uma tarefa que ele próprio diz não conclusa. O ponto de referência para esta discussão é sempre a ideia de que o social só pode ser pensado e discutido na própria sociedade, o único locus do social.

Esses questionamentos "perturbaram" diversos intelectuais. Na Alemanha, a resistência inicial com a Teoria foi significativa, assim como em outros países da Europa. Contudo, o reconhecimento europeu da importância da Teoria ocorreu e, hoje, os textos de Luhmann estão traduzidos em muitas línguas. Na América Latina, no Chile, Dario Rodrigues foi um dos primeiros a estudar profundamente essas indagações; no México, Javier Torres Nafarate. Ambos tornaram-se os mais importantes tradutores das obras de Luhmann, permitindo que os intelectuais de língua hispânica pudessem conhecer a Teoria dos Sistemas. Atualmente temos uma "Escola Sistêmica" consolidada na América Latina. Alguns seguem estudando os "desassossegos" da Teoria, como Osvaldo Dallera, na Argentina, que nos auxilia a refletir por que a Teoria continua a "molestar" tanto:

La sociología de Luhmann molesta porque (...) interpela, desafía y pone en aprietos al pensamiento tradicional e sus variantes pre modernas y modernas. ¿Cómo hace la sociología de Luhmann para molestar y ser irritante? Básicamente hace tres cosas: (...) Desplaza del centro de la escena social a las autoridades del pensamiento tradicional; (...) Hace de la observación el motor de la construcción social de la realdad; (...) Asume que todo lo que no es social pasa a formar parte de todo lo demás que se agrupa en el campo ecológico ${ }^{5}$. (Dallera, 2012, p.131)

\footnotetext{
4 Falamos aqui das teorias marxistas de uma maneira geral. Sabemos, contudo, que existem pensamentos estruturados na doutrina marxista que sustentam a possibilidade de diferenciação entre sujeito-objeto, porém tal explanação desviaria o foco da presente discussão.

5 "A sociologia de Luhmann molesta porque (...) interpela, desafia e põe em apertos o pensamento tradicional e suas variantes pré-modernas e modernas. Como faz a sociologia de Luhmann para molestar e ser irritante? Basicamente faz três coisas: (...) Retira do centro de cena social as autoridades do pensamento tradicional; (...) Faz da observação o motor da construção social da realidade; (...) Assume que tudo que não é social passa a pertencer a determinado agrupamento no campo ecológico." Tradução Livre.
} 
Tem razão Dallera em utilizar a expressão "molesta". Para muitos, basta ouvir o nome do autor que já se sentem molestados, mas esse molestamento não tem feito com que grande parte dos mesmos críticos à Teoria leiam o autor, pois, além de molestar, a Teoria Sistêmica desconstrói pressupostos que sempre consideramos válidos e propõe constantemente uma nova reflexão, ou melhor, uma nova observação da própria observação. Isso desacomoda qualquer leitor que buscar respostas imediatas e definitivas.

A limitação que encontramos (que também, ou exatamente por isso, é igualmente possibilidade) é que todas as vezes que refletimos sobre o sistema médico, não encontramos o conceito de saúde ampliado. Encontramos apenas a saúde como mera ausência de doença, conceito esse que não é compatível com a proposta do projeto de investigação que estamos desenvolvendo, pois partimos do pressuposto de que saúde, em primeiro lugar, é um bem da comunidade (Saccheri; Massulo; Mangone, 2008, p. 40-49). Em segundo lugar, concordamos com o conceito definido pela Organização Mundial da Saúde e também com a ideia de que para definir saúde é preciso entender os determinantes sociais da saúde. Essas são nossas dificuldades, com as quais não buscamos - e mesmo que buscássemos, não encontraríamos - respostas ou certezas, mas a compreensão dessa nova situação desde a Teoria Sistêmica.

Luhmann (Luhmann; De Giorgi, 1996), quando estudou o sistema médico, não tinha a preocupação com a dimensão que estamos dando para o conceito de saúde e para o direito ao direito à saúde. Ele descreveu o sistema tal como se apresentava, dizendo, inclusive, que o sistema médico só tem sentido quando opera com o código negativo, ou seja, com a doença. Esse fator nos inquieta de modo constante e tem sido objeto de várias discussões em seminários, encontros e colóquios. O desafio atual é, cada vez que procurarmos o sistema da medicina, encontrarmos o sistema da saúde e não o contrário, que é o que vem acontecendo.

Essa limitação é nosso atual desassossego. Muito temos discutido sobre a saúde como um sistema social, discussão iniciada há mais de dez anos, com vários pensadores da Teoria Sistêmica, em especial com Giancarlo Corsi. Assim, o desafio que nos propomos é reduzir a complexidade, mostrando que a saúde é um sistema, 
embora saibamos que reduzir a complexidade é sempre, ao mesmo tempo, incrementar a complexidade ${ }^{6}$.

No caso específico do sistema da saúde, temos de revistar várias teorias e disciplinas para darmos conta da tarefa, pois cada vez que definimos um conceito, cada vez que observamos, fazemos isso estabelecendo distinções, indicamos alguma coisa diferenciando-se de outras. Por isso, assim transitamos na perspectiva construtivista: construímos e desconstruímos constantemente distinções, fruto de observação de várias ordens, fruto de uma complexidade que apresenta várias dificuldades e, que muitas vezes, tem de ser definida de forma tautológica.

\section{Saúde: um sistema diferenciado funcionalmente}

Attraverso la differenziazione del sistema, in una certa misura, il sistema se moltiplica in se stesso mediante distinzioni sempre nuove tra sistema e ambienti nel sistema ${ }^{7}$. (Luhmann; De Giorgi, 1996, p.249)

Se o conceito de diferenciação funcional foi tema de desconforto, hoje é um dos conceitos mais aceitos pela academia, ou seja, a ideia de que a democracia só é possível em sociedades diferenciadas funcionalmente é ponto relativamente pacífico.

Mesmo sabendo que a democracia tem na sua possibilidade também a sua limitação, as diferenças entre os mais diversos sistemas sociais é extremamente importante para que se possa pensar na realização de uma democracia, isso porque somente em uma sociedade diferenciada funcionalmente podemos efetivar a "soberania popular", inclusive questionando-a. Só desse modo vamos entender como a democracia encobre, muitas vezes, o poder ou, em alguns casos, é encoberta pelo poder, o que também possibilita a diferença entre o sistema da política, do direito, da educação e de todos os sistemas sociais diferenciados.

\footnotetext{
6 "Complessità non è un'operazione che un sistema effettua o che in esso si verifica: complessità è un concetto dell'osservazione e della descrizione. Dobbiamo chiederci, allora: qual è la forma di questo concetto, qual è la distinzione che lo costituisce? Già questa domanda porta ad un infinità di riflessioni che vi si raccordono, perchè il concetto di complessità non è un concetto semplice, ma è un concetto a sua volta complesso e quindi formato in modo autológico". (Luhmann; De Giorgi, 1996, p. 40). "A complexidade não é uma operação que um sistema efetua ou que nele se verifica: complexidade é um conceito da observação e da descrição. Devemos nos perguntar, então: qual é a forma deste conceito, qual é a distinção que o constitui? Já este questionamento leva a uma infinidade de reflexões que se imbricam, porque o conceito de complexidade não é um conceito simples, mas é um conceito por sua vez complexo e, então, formado de modo autólogo". Tradução Livre.

7 "Por meio da diferenciação do sistema, até certo ponto, o sistema se multiplica em si mesmo sempre mediante novas distinções entre sistema e ambiente no sistema". Tradução livre.
} 
A sociedade como um sistema social é composta por subsistemas, é constituída por comunicação, ou seja, é uma malha de comunicações. Sem comunicação, não é possível fazer nenhuma seleção, escolha. A necessidade de seleção (escolha) decorre justamente do fato de o sistema não conseguir dar conta desse contingente de possibilidades, da complexidade interna. Esse excesso de possibilidades é proporcional à gama de elementos do seu interior, e as relações entre esses elementos fazem crescer o número de possibilidades. Esse crescente número de possibilidades torna a sociedade altamente complexa, de risco e em permanente evolução.

É exatamente esse processo evolutivo que permite a constante diferenciação funcional, na qual as possibilidades não realizadas ficam potencializadas como opções para o futuro e, de algum modo, são absorvidas pelo sistema.

O sistema seleciona algumas possibilidades, opções que estejam em consonância com a função que ele desempenha. É a simplificação da complexidade do ambiente como condição de sobrevivência do sistema, mas isso desencadeia o aumento da sua complexidade. O número de possibilidades aumenta internamente, podendo até gerar sua autodiferenciação, resultando em novos subsistemas ${ }^{8}$. O motivo que enseja a sua evolução é a sobrevivência à complexidade, que cria constantemente novas possibilidades. Ou seja, na tentativa de reduzir a complexidade, dá-se o incremento dela. Diante disso, também podemos afirmar que a saúde se constitui em um sistema social diferenciado funcionalmente.

A dificuldade de alguns com esse entendimento está vinculada - pelo menos em alguns casos - ao fato de que Luhmann nunca escreveu nada sobre o sistema da saúde, mas sim sobre o sistema médico. No entanto, acreditamos que a Teoria ofereça elementos suficientes para afirmarmos que, no processo evolutivo, a saúde foi aprimorando suas comunicações e estruturando-se como um subsistema social, ou em sistema social.

\footnotetext{
${ }^{8}$ Para elucidar tal situação, tome-se, como exemplo, o caso do Direito: inicialmente o sistema do direito diferenciou-se em público e privado. Entretanto, dada sua crescente complexidade, viu-se obrigado a autodiferenciar-se em constitucional, administrativo, penal, comercial e, assim, sucessivamente. Esse processo revela a evolução do sistema, que é diferente de 'desenvolvimento', que é passível de controle, enquanto a evolução não é controlável. (Luhmann, 1983).
} 
Seguindo a própria definição de sistema social, vemos que a saúde se enquadra perfeitamente bem no conceito de sistema social, pois surge por "autocatálise" dos problemas de dupla contingência. (Luhmann, 2007)

Ainda, um sistema social precisa diferenciar-se de seu ambiente e ter uma estrutura própria, ser fechado operativamente e aberto cognitivamente. Aliás, somente esse fato pode justificar a ideia de irritação entre o sistema do direito e o sistema da saúde, os dois sistemas fechados, mas em constante irritação, especialmente em países da periferia da modernidade, nos quais o direito é constantemente chamado a decidir sobre questões "não decididas" por outros subsistemas.

O que faz o sistema do direito é processualizar as informações que vêm de outros sistemas por meio da sua linguagem interna, do seu código e da sua estrutura, ou seja, quando algum sistema irrita outro, temos um processo normal e necessário para a própria evolução. O que não pode acontecer é que os sistemas irritados decidam com códigos diferentes dos seus.

Em outros termos: os sistemas sociais que não decidem com seus códigos estão provocando uma corrupção, o que não é adequado, pois quando um sistema decide com o código do outro, perdemos a possibilidade da diferenciação funcional. Os reflexos desta eventual "corrupção" são percebidos, por exemplo, através do déficit democrático que temos atualmente.

Vemos que, em todas as sociedades, o sistema da saúde foi se diferenciando e se autorreproduzindo, especializando suas comunicações, diferenciando-se por meio da contínua irritação com os demais sistemas sociais.

Atualmente discutimos muito a relação entre saúde e direito, mas não é apenas essa relação que observamos em constante crescimento: temos uma constante irritação entre sistema da saúde e sistema da política, outro indicador importante da saúde como sistema social.

Podemos observar que o sistema da saúde se diferencia dos demais sistemas sociais, tem uma estrutura própria, opera com um código binário, ainda que o valor que ative o sistema não seja o valor positivo - a saúde -, mas sim a doença, ou seja, o código negativo. 


\section{Sistema Médico}

Il sistema dela medicina o sistema di cura dei malati è uno dei sistemi parziali per della società differenziata per funzioni. Questo sistema si orienta primariamente all' ambiente della soceità, è concentrato sui problemi che osserva in tale ambiente: la comunicazioni al suo interno non si occupa di se stessa, bensi delle condizioni organiche o mentali degli uomini'. (Corsi, 1996, p.213).

Se, para Luhmann, o sistema médico tem uma estrutura própria, vemos que, por meio dessa observação, é possível pensar no sistema da saúde. No entanto, não podemos desconsiderar as observações do autor sobre o sistema médico, ou melhor, sobre medicina, pois ele afirma que o "sistema da medicina" é igual ao sistema da "cura dos doentes", o que atualmente pode ser questionado, pois não está claro na Teoria Sistêmica qual o conceito de saúde utilizado.

Hoje é senso comum entender a saúde muito além da "cura dei malati". Ora, se assim entendemos, obviamente outra compreensão é possível ter desse sistema.

Interessante observar que Luhmann escreveu poucos artigos sobre o sistema médico, nos quais a saúde aparece sempre como parte do código binário saúde/doença, ou melhor: do código são/doente. Alerta Luhmann, no entanto, que os conceitos de são e de doente não indicam um particular estado físico ou psíquico, mas têm o valor de código, no qual o valor positivo é o da doença e o negativo é o da saúde. Ou seja, o que vai importar para esse sistema não é a saúde, mas sim a doença, já que só essa importa para os médicos.

Para quem não entende a perspectiva da Teoria isso pode parecer absurdo, porém é fundamental destacar o que o autor quer dizer com isso: para os médicos, a questão a ser resolvida é a doença, não a saúde:

[...] non à niente da fare, consente solo di riflettere su ciò che viene a mancare quando si è malati. II sistema della medicina opera, dunque, soltanto quando qualcuno si ammala. Corrispondentemente, vi sono molti malatia ed una sola salute: la terminologia della malattia si sviluppa, mentre II concetto di salute rimane problematico o vuoto ${ }^{10}$. (Corsi, 1996, p.143).

\footnotetext{
9 "O sistema da medicina ou sistema de cura dos doentes é um dos sistemas parciais de uma sociedade diferenciada funcionalmente. Esse sistema é voltado principalmente ao ambiente da sociedade, com foco nos problemas que se observam em tal ambiente: a comunicação interna não se ocupa de si mesma, mas das condições orgânicas ou mentais dos homens." Tradução livre.

10 “[...] não dá para fazer nada, vamos apenas pensar sobre o que é perdido quando o indivíduo está doente. O sistema médico opera, então, só quando alguém fica doente. Do mesmo modo, há muitas doenças e uma só saúde: a terminologia da doença se desenvolve, enquanto o conceito de saúde continua a ser problemático ou vazio." Tradução livre.
} 
Ora, se o sistema da medicina opera somente quando alguém adoece, estamos distantes do conceito de direito à saúde constitucionalizado em grande parte dos países, ou ainda podemos concluir que o autor já se referia às dificuldades de efetivar e dimensionar a própria definição da Organização Mundial da Saúde sobre saúde, bem como as especificidades da saúde em cada comunidade.

Outro aspecto relevante é que o valor significativo para o sistema da medicina não é o valor positivo (estar são), mas sim a doença, já que o sistema da medicina só opera orientado para a doença. Aqui temos mais uma razão para pensar no sistema da saúde, no qual o médico não atua como única figura. Em outros termos:

I programmi della medicina si producono soltanto nel contesto della codificazione sano/malato e, quindi, sono orientati alla malattia [...] Poiché II suo valore positivo è la malattia, la medicina non ha neppure costruito una teoria della reflessione. L'agire medico deriva dal valore attribuito alla salute: ma la salute non crea collegamenti nel sistema e, dunque, non c'è nulla su cui riflettere ${ }^{11}$. (Corsi, 1996, p.144).

Com estas observações, podemos ver que Luhmann deixa indicativos para se pensar na saúde como um sistema social, em cujo contexto ele destaca a existência do código binário referido, mas alerta para a constatação de que o valor que "vale" é o negativo, ou seja, a doença: ela é que faz o sistema "funcionar" e ser funcionalmente diferenciado dos demais sistemas sociais, mas sempre conectado nessa rede de comunicação que é a própria sociedade, como podemos observar:

La interdipendenze tra medicina ed altri sistemi di funzioni sono molto importanti. Il sistema della medicina è strutturalmente accoppiato con I' economia, la scienza, II sistema giuridico e cosi via: la cura medica richiede decisioni politiche, conoscenze scientifiche, finanziamenti, regolazione giuridica. La interdipendenze non intaccano l' autonomia del sistema della medicina: possono essere coinvolti uffici di lavoro, sedute parlamentari, comissioni etiche, preti, parenti, ma la costruzione della malatia rimane matéria della medicina ${ }^{12}$. (Corsi, 1996, p.145).

\footnotetext{
11 "Os programas da medicina se produzem apenas no contexto da codificação saudável/doente e, por conseguinte, são orientados para a doença [...] porque o seu valor positivo é a doença, a medicina não tem sequer construído uma teoria da reflexão. $O$ ato médico deriva do valor atribuído para a saúde, mas a saúde não cria ligações no sistema e, portanto, não há nada para refletir." Tradução livre.

12 "As interdependências entre medicina e outros sistemas de funções são muito importantes. O sistema médico é estruturalmente acoplado com a economia, a ciência, o sistema legal e assim por diante: a assistência médica requer decisões políticas, conhecimentos científicos, financiamento, regulamentação legal. As interdependências não afetam a autonomia do sistema da medicina: podem estar envolvidos escritórios de trabalho, sessões parlamentares, comissões de ética, padres, familiares, mas a construção da doença continua a ser uma matéria da medicina." Tradução livre.
} 
Aqui temos mais indicativos da possibilidade, independentemente de Luhmann ter descrito a saúde como sistema social. Além disso, ele aponta para o caráter transdisciplinar e intersetorial da saúde, mesmo afirmando que doença é matéria da medicina.

Para os sanitaristas, essa abordagem pode render críticas, porém essa não é nossa preocupação: queremos mostrar que o autor deixa instrumentos suficientes para o entendimento da saúde como um sistema diferenciado funcionalmente.

Temos outra passagem interessante que nos leva para esse caminho: aquela na qual Luhmann afirma que o sistema médico pode ter também um código secundário:

Si, afferma, invece, la possibilità di un códice secondario, grazie alla tecnologia genética: la distinzione geneticamente perfetto / geneticamente preoccupante permette di determinare una distinzione secondaria curabile / incurabile, riferita alla malattia: la cura dei malati viene poi richiesta su entrambi i lati della distinzione ${ }^{13}$. (Corsi, 1996, p.146).

Ou seja, Luhmann (Luhmann; De Giorgi, 1996) percebeu a complexidade que envolve o sistema da medicina e deixa espaço para novas reflexões que ultrapassam os campos médico, ético e político. Com isso, mostra que, evolutivamente, o campo da medicina vem se ampliando, motivado pela inflação das possibilidades de tratamento, bem como pelos seus custos.

Sabe-se que a imutabilidade não é característica dos sistemas, visto que o ambiente é efetivamente complexo, de modo que o influencia internamente. Com a saúde, isso não foi diferente, especialmente se pensarmos na diferenciação funcional do Direito até chegarmos ao direito à saúde.

Deve-se dizer que a diferenciação do sistema não significa decomposição de um todo em partes, mas sim que cada subsistema tem seu próprio entorno. Não existe um agente externo que modifica: é o próprio sistema que, por uma questão de sua sobrevivência no ambiente, realiza essa diferenciação. Justamente em face dessa mutabilidade, ele foi capaz de autoproduzir-se e, assim, alcançamos a expectativa jurídica da saúde. Ele foi irritado suficientemente para fazer a sua seleção

\footnotetext{
13 "Se afirma, no entanto, a possibilidade de um código secundário, graças à tecnologia genética: a distinção do geneticamente perfeito/geneticamente preocupante nos conduz a determinar uma distinção secundária curável/incurável, referida à doença: o tratamento e a cura dos pacientes são, então, necessários em ambos os lados da distinção". Tradução livre.
} 
e, dentro dessa, a seleção da saúde com o intuito de torná-la expectativa jurídica (regra).

A evolução dos sistemas não ocorre de modo isolado, pois ela depende de irritações do ambiente, e essas irritações, levando em consideração a tolerância do sistema, podem fazê-lo mudar suas estruturas, ou seja, produzir a si mesmo. Sistemicamente, é o que se pode denominar de autopoiese ${ }^{14}$. A autopoiese, por sua vez, é responsável pelo aumento constante das possibilidades até que a complexidade atinja um nível extremamente elevado, nível esse não suportado pela estrutura do sistema, levando-o a diferenciar-se.

O direito diferenciou-se, sofreu irritações do ambiente ao ponto de autoproduzir-se no tocante à saúde como direito, visto que, a partir das irritações do ambiente, ele processou-as conforme o seu código (binário) e positivou, reconheceu a saúde como direito.

Essas irritações foram oriundas, especialmente, do Movimento Sanitário, correspondente à luta pela efetivação do direito à saúde e por uma saúde pública e acessível a todos, e de toda a lógica que permeou a mudança de paradigma na saúde: a saúde já não era mais um mero ato de caridade ou assistencia; passa a ser um direito constitucionalizado.

Esses aportes teóricos no tocante à constitucionalização do direito à saúde servem para compreendermos a evolução do sistema do direito. A Constituição de 1988, com a positivação do direito à saúde, é fruto de uma malha de comunicações que confluíram para uma finalidade: reconhecer o direito à saúde na via constitucional. O sistema do direito recebeu diversas irritações, tais como aquelas advindas do Movimento Sanitário, que podem ser observadas nos fatores mencionados no começo da análise constitucional. Com a institucionalização dos direitos fundamentais, em especial o direito à saúde, a Constituição acabou por reconhecer a grande complexidade do processo efetivação desse direito e, a partir disso, pretendeu responder aos anseios (exigências) do ambiente, por intermédio da comunicação produzida para que isso ocorresse (Neves, 2007, p. 74-75).

\footnotetext{
${ }^{14}$ Foi assim denominada pelos biólogos Maturana e Varela. Por ser uma teoria transdisciplinar, Luhmann trabalhou também com biologia, matemática e física.
} 


\section{Ponderações finais}

Un sistema sociale è un sistema autoreferenziale autopoietico, che si costituisce come diferenza rispetto ad un ambiente ${ }^{15}$. (Corsi, 1996, p.40).

A construção teórica da saúde como um sistema social, em uma sociedade em permanente transformação, é possível, embora apresente algumas limitações, as quais serão superadas através do paradoxo limite/possibilidade.

O modo da Teoria dos Sistemas de operar de forma transdisciplinar, construtivista, nos leva para o caminho da transformação social, com a qual, muito mais do que uma mudança que não altere determinada situação estruturalmente ou uma reforma em que os reformadores nunca são reformados, optamos por trabalhar com o conceito de transformação, pois este também rompe com a ideia da separação sujeito-objeto, uma vez que, ao transformarmos a sociedade, transformamos o ambiente dessa sociedade.

Várias são as opiniões dos filósofos anteriores: mudança como avanço, como regresso, como resultado de contradição. Luhmann não adota um ou outro pensamento; adota uma postura que, de certa forma, compila todas essas formas de pensamento.

Reações e manifestações provocam transformações no sistema social, o que indica que é preciso olhar o passado para produzir novas alternativas. Ou seja, quem decide leva em consideração o passado, a opinião pública, os movimentos de protesto. A decisão não é tomada por nenhum destes elementos; é tomada dentro da estrutura em que está inserida. Da mesma forma que, por ser tomada dentro dessa estrutura, será também objeto de oposição, muitas vezes dos próprios grupos que contribuíram para aquela tomada de decisão, justamente porque, ao decidir, toma-se partido de uma ou outra possibilidade de decidir.

O problema está quando a decisão não é tomada dentro das estruturas, ou seja, pelas instituições que a compõem, tornando seus efeitos ainda mais imprevisíveis. As estruturas não conduzem mudanças ou não mudança ${ }^{16}$, mas estas devem se dar através delas.

\footnotetext{
15 “Um sistema social é um sistema autorreferencial autopoiético que se constitui como diferença em relação a um ambiente". Tradução livre.

16 "I mutamenti di struttura nel sistema e i mutamenti nell'ambiente hanno luogo senza coordinamento; se dovessero essere coordinati, ci si dovrebbe preoccupare ad hoc per le casualità che ne deriverebbero." (Luhmann, 1996, p. 273) "As mudanças de estrutura no sistema e as mudanças no
} 
O processo de mudança social chamado "endógeno-exógeno", se observado no longo prazo e, desenvolvendo-se, produz resultados que podem afetar não somente as regras de funcionamento do sistema em que aparecem, mas também o entorno do sistema, provocando uma reação desse último.

Neste artigo, também apresentamos a preocupação com alguns conceitos utilizados de forma "banal" e, muitas vezes, relacionados com a Teoria Sistêmica. É o caso da expressão "judicialização", que reporta a uma série de reflexões: a) o direito à saúde é entendido como direito à assistência sanitária, e esse é um sinal típico da tendência de inclusão generalizada, assim como ocorre com o direito à educação e a votar; b) imposição de atendimento à saúde por parte de um tribunal, o que revela um sério problema também para o sistema econômico, pois não existe verba suficiente para o atendimento pleno da saúde. Além disso, um juiz pouco sabe (e nem tem de saber) de saúde; então, quando impõe uma terapia, pode cometer erros graves, o que não significa que o direito não possa obrigar o sistema da saúde a atender alguém, o que não pode é dizer qual terapia é necessária; c) como consequência desses itens acima, temos: os problemas de saúde são tratados pelo direito, fazendo com que a economia "obrigue" a política a intervir.

Assim, todos os sistemas se agitam (se irritam, autorreproduzem, geram mais diferenças: em síntese, evoluem), e as pré-teses aparecem, mas o sistema da saúde continuará operando do modo como opera. Em outros termos: irá operar com os recursos financeiros disponíveis, esperando que alguém imagine uma possível reforma no sistema e, depois, uma reforma (ou não) dos reformadores.

Enfim, sistema da saúde ou da medicina? Para Luhmann (Luhmann; De Giorgi, 1996), interessou somente esclarecer o que é a função da medicina e, por isso, tratou somente desse sistema. A medicina cura as doenças e constrói também, mas não produz saúde porque esta não existe. É necessário considerar que os códigos binários (doença/saúde, direito/não direito etc.) não indicam necessariamente a situação da realidade, mas indicam comunicação: quando um juiz dá razão a uma parte e não à outra, não afirma que a outra parte poderia não ter razão, diz somente que deve decidir e, assim, demarca no sentido positivo ou negativo a própria decisão.

ambiente têm seu lugar sem que haja uma coordenação; se devessem ser coordenadas, deveriam se preocupar ad hoc com a casualidade que delas derivaria". Tradução Livre. 
Do mesmo modo, quando um profissional da saúde diz que alguém não tem uma doença, não afirma que tal pessoa é completamente sã, diz somente que não encontra motivos para intervir e, assim, marca a sua decisão, ou o contrário, naturalmente. Nesse sentido, o valor do código que tem cognitividade é aquele da doença, independentemente da preferência do paciente, que é ter saúde.

\section{Referências}

CORSI, Giancarlo. Sistemi meddici. Lecce :1996 (mimeo).

CAMPILONGO, Celso F. Interpretação do Direito e Movimentos Sociais. Rio de Janeiro : Elsevier, 2012. ISBN 9788535254105

DALLERA, Osvaldo. La sociedad como sistema de comunicación. La Teoría Sociológica de Niklas Luhmann en 30 lecciones. Buenos Aires : Editorial Biblos, 2012. ISBN 9789507867860

LUHMANN, Niklas. La sociedad de la sociedad. (1. ed.) México: Herder, 2007. ISBN: 9789685807203

LUHMANN, Niklas; DE GIORGI, Raffaele. Teoria della società. (8 ed.) Milão : Franco Angeli, 1996. [ISBN $\mathrm{n}$. fornecido]

LUHMANN, Niklas. Sociologia do Direito I. Trad. Gustavo Bayer. Rio de Janeiro: Tempo Brasileiro, 1983. [ISBN n. fornecido]

NEVES, Marcelo. A constitucionalização Simbólica. São Paulo : Martins Fontes, 2007. ISBN 9788560156399

SACCHERI, Tullia; MASULLO, Giuseppe; MANGONE, Emiliana. Sociologia della Salute: Fondamenti e Prospecttive. Mercato San Severino : C.E.I.M. Editrice, 2008. ISBN 9788895304045 